\title{
Skeletal Muscle Channelopathies
}

Vinojini Vivekanandam MBBS ${ }^{1}$, Pinki Munot MRCPCh${ }^{2}$, Michael G Hanna FMedSci ${ }^{1}$, Emma Matthews $\mathrm{MRCP}^{1}$

${ }^{1}$ Queen Square Centre for Neuromuscular Diseases and Department of Neuromuscular Diseases, Queen Square Institute of Neurology, UCL and National Hospital for Neurology and Neurosurgery, London, WC1N 3BG, UK

${ }^{2}$ Dubowitz Neuromuscular Centre, Great Ormond Street Hospital for Children, London, UK.

Vinojini.vivekanandam@nhs.net

m.hanna@ucl.ac.uk

\section{Pinki.munot@gosh.nhs.net}

\section{emma.matthews@ucl.ac.uk}

Corresponding author: Emma Matthews

Disclosures: Part of this work was undertaken at University College London Hospitals/University College London, which received a proportion of funding from the Department of Health's National Institute for Health Research Biomedical Research Centres funding scheme. MGH receives research funds from the Medical Research Council and the UCLH Biomedical Research Centre. EM receives research funds from Wellcome..

Keywords: Myotonia, Myotonia Congenita, Paramyotonia Congenita, Periodic Paralysis, Channelopathies

\section{Key Points}

- There are several conditions to consider in patients displaying myotonia on electromyography (EMG). Myotonic Dystrophy type 1 is the most common differential diagnosis.

- Determining a genetic diagnosis and inheritance pattern is essential for informing family planning. 
- Hypokalaemic periodic paralysis can be more severe in males and require polypharmacy to control symptoms.

- Accurate diagnosis is important to inform effective treatment options available for skeletal muscle channelopathies.

- Cardiac conduction defects seen in Andersen-Tawil Syndrome require monitoring and involvement from cardiac colleagues, even if asymptomatic.

- The skeletal muscle channelopathies can have significant impact on quality of life. Beyond myotonia and paralysis, pain and fatigue are important aspects to consider in management.

\section{Synopsis}

Skeletal muscle channelopathies are rare genetic neuromuscular conditions that include the nondystrophic myotonias and periodic paralyses. They cause disabling muscle symptoms and can limit educational potential, work opportunities, socialisation and quality of life. Effective therapy is available making it essential to recognise and treat this group of disorders. Here we highlight important aspects regarding diagnosis and management using illustrative case reports. 


\section{Introduction}

Skeletal muscle channelopathies are a group of rare heterogeneous neuromuscular disorders characterised by episodic disabling symptoms of either myotonia or paralysis. They are genetic disorders, and onset is predominantly in childhood. Symptoms can have a significant impact on quality of life, and influence educational opportunities, employment choice and pregnancy. A subset develop fixed myopathy with progressive disability. They are important disorders to recognise and diagnose as symptomatic treatment is available and can make a significant difference to quality of life and morbidity.

Estimated minimum point prevalence of the skeletal muscle channelopathies is approximately 1.12 per $100000^{1}$. With increased awareness, and the advent of next generation sequencing, prevalence is now likely to be higher. The skeletal muscle channelopathies are broadly divided into the nondystrophic myotonias and the periodic paralyses. The non-dystrophic myotonias include myotonia congenita (MC), paramyotonia congenita (PMC) and sodium channel myotonia (SCM). Myotonia is often experienced by patients as stiffness, cramps and locking which may be painful. Myotonia congenita, due to mutations in the CLCN1 gene is the most common of all the skeletal muscle channelopathies ${ }^{1}$. Myotonia predominantly affects the legs and falls can be common. The hallmark of this condition is the warm up phenomenon (improved myotonia with repetition). Paramyotonia congenita due to mutations in the SCN4A gene typically affects the hands and eye/facial muscle more than the legs. Paradoxical myotonia is demonstrated (worsening of myotonia with repetition). Episodes of weakness or even frank paralysis may occur in PMC. Sodium channel myotonia, also due to mutations in the SCN4A gene, has a similar distribution of muscle involvement to PMC but both warm up and/or paradoxical myotonia may be found. These are purely myotonic phenotypes without any episodic weakness. All of the NDMs can be exacerbated by cold, but it is most striking in PMC, exercise or prolonged rest. Muscle hypertrophy can also present in any NDM although is typically highlighted in MC. 
The periodic paralyses include hyperkalaemic periodic paralysis due to mutations in the SCN4A gene $^{2,3}$, hypokalaemic periodic paralysis due to mutations in the CACNA1S gene (up to $80 \%$ ) and the SCN4A gene ${ }^{4}$ and Andersen Tawil Syndrome (ATS) due to mutations in the KCNJ2 gene ${ }^{5}$. ATS is the only skeletal muscle channelopathy to involve tissues other than skeletal muscle, namely the heart (cardiac conduction defects) and bone development (dysmorphic features) ${ }^{6}$. During an attack of paralysis, patients experience disabling weakness and areflexia. Between attacks, muscle strength is often normal. Triggers can include exertion, carbohydrate rich meals, cold and stress.

We illustrate important clinical aspects regarding diagnosis and management of the skeletal muscle channelopathies with the use of five clinical cases.

\section{CASE A: An Alternative.}

A 62 year old female presented with a history of muscle stiffness and myalgia since childhood. Past medical history included a previous hysterectomy for menorrhagia, hypercholesterolemia and fatty liver.

Early history and development was normal but at the age of eight she recalls that her feet would often be 'stuck' when skating on ice. At age 21 , when playing rounders, she fell over when trying to run after hitting the ball. Symptoms worsened during each of her pregnancies and climbing stairs became difficult. Her symptoms could be exacerbated in cold environments, and her condition deteriorated when she was prescribed a statin.

In her fifties, she noted the development of other symptoms. She felt unbalanced if standing unaided for long periods. Significant exercise left her fatigued the following day. She enjoyed hiking but found stepping over boulders very difficult even with walking poles. She lost the ability to jump. In terms of family history, her two daughters reported difficulty opening jars and similar muscle stiffness during pregnancy. Her mother and sister had apparently reported "difficulty with cold hands and feet" but were not evaluated in our clinic. 
On examination at the time of referral in her fifties she had hand grip myotonia (video 1). Some mild proximal weakness was detected - medical research council (MRC) grade 4+ in the shoulder abductors and hip flexors. CK was $418 \mathrm{IU} / \mathrm{L}$. Electromyography demonstrated myotonia and a positive short exercise test with Fournier type III pattern. This pattern of short exercise test is nonspecific and can be present in sodium channel myotonia, autosomal dominant myotonia congenita and myotonic dystrophy type 2. Given the lack of systemic features she was diagnosed with a nondystrophic myotonia on clinical grounds. There was no history of cardiac disease or symptoms, ECG was normal and she commenced Mexiletine with significant symptomatic improvement.

Genetic testing identified a novel variant in the SCN4A gene (c.4805A>G, p.Asn1602Ser) that was present in the proband and one of her affected daughters who we had also assessed and confirmed to have a myotonic disorder. Her second daughter who also reported symptoms tested negative for this mutation. This discrepancy raised doubt as to the pathogenicity of the SCN4A variant. We recommended she be assessed in her local centre as she lived abroad, and undergo an EMG. In parallel with this, additional lab-based functional testing to determine electrophysiological evidence of pathogenicity of this novel gene variant was commenced.

Lab based assessment showed the variant had no significant effect on channel function indicating it was a benign variant. The daughter's EMG demonstrated she had myotonia confirming that she was affected and therefore the SCN4A variant did not segregate with disease in the family. Genetic testing for myotonic dystrophy type 2 was requested in the proband and was positive with a CCTG repeat in intron1 of the CNBP gene. This diagnosis was subsequently confirmed in both her daughters.

On the day of the proband's clinic appointment to discuss the new genetic results she attended clinic and reported she had remained generally well but had just been diagnosed with cataracts.

\section{Video 1: hand grip myotonia seen in myotonia congenita.}


What is the most common diagnosis in a patient presenting with myotonia?

1. Myotonia congenita

2. Paramyotonia congenita

3. Myotonic dystrophy type 2

4. Pompe's disease

5. Myotonic dystrophy type 1

Correct answer: 5

Discussion

\section{Differential diagnosis of myotonia}

Myotonia is delayed relaxation of skeletal muscle after voluntary contraction. It is often described by patients as muscle stiffness, cramp or 'locking'. Symptoms commonly affect the hands and legs but can involve any skeletal muscle including those of the face, jaw, tongue and trunk. In some patients, it can even affect intercostal muscles causing dyspnoea. Myotonia is best examined with initiation of gait, in the hands and eyes (Video 2). In Myotonia congenita, myotonia lessens with repetition whereas in paramyotonia, myotonia worsens with repetition (Video 3).

Myotonia can be present in several conditions including the non-dystrophic myotonias and myotonic dystrophy type one and two. DM1 is the most common myotonic disorder with an incidence of 1 in 8000 and although typically accompanied by a degree of distal weakness and/or extra-muscular manifestations such as cataracts, diabetes, frontal balding and testosterone deficiency these features may not yet be evident early in the disease course (or at the age when NDM typically presents) ${ }^{7}$. Extra-muscular features are likewise reported in DM2 along with proximal weakness but may be mild. It is always important to consider a differential diagnosis of myotonic dystrophy in patients with myotonia as the systemic and cardiac features require a different monitoring and management approach to the skeletal muscle exclusive non-dystrophic myotonias. 
Electrical myotonia, often without overt clinical myotonia can also be seen in other neuromuscular disorders including Acid Maltase deficiency (Pompe's disease), myopathies including myofibrillar myopathies, denervation, drug-induced hypothyroidism, colchicine induced myopathy ${ }^{8}$.

\section{Video 2: eyelid myotonia}

\section{Video 3: paradoxical myotonia in paramyotonia congenita.}

\section{Differentiating NDM from myotonic dystrophy}

Obvious systemic features associated with myotonic dystrophy (DM) versus an isolated myotonic disorder generally assist in differentiating these diseases although the caveats above of early disease course in DM1 and milder symptoms in DM2 can cause confusion (as in our described family). Myalgia is often suggested as a pointer towards DM2 but it is not specific and can be significant among the NDMs as well. The short exercise test with variable patterns as described by Fournier can be indicative if characteristic (patterns 1 and 2) but as in our case pattern 3 is non-informative ${ }^{9}$. Genetic testing is ultimately diagnostic although the possibility of polymorphisms in a candidate gene can be misleading (as in our case). It is also notable that single heterozygous CLCN1 gene mutations and DM2 can co-exist ${ }^{10}$ or can modify a sodium channel phenotype ${ }^{11}$.

\section{Is it safe to use mexiletine to treat myotonia in myotonic dystrophy?}

Our proband derived significant benefit from taking the class I anti-arrhythmic sodium channel blocker mexiletine. In the NDMs i.e. those without pre-existing heart disease it has a good safety profile and this is largely considered to be first line therapy. It can be arrythmogenic however and this raises greater concern in myotonic dystrophy due to the risk of pre-existing cardiac disease. Atrioventricular and interventricular conduction defects are common ${ }^{12}$. Additionally, approximately $14 \%$ have left ventricular systolic dysfunction. In a trial of mexiletine in patients with myotonic dystrophy, no significant difference was seen in ECGs and cardiac events between mexiletine and placebo groups ${ }^{13}$. However, patients with significant cardiac arrhythmias were excluded from this 
study. Given the theoretical arrhythmogenic risk with mexiletine, caution should be taken when using it in patients with myotonic dystrophy and associated cardiac disease.

Our patients had normal ECGs performed as part of routine monitoring while on mexiletine and reported no symptoms suggestive of cardiac disease. Following the diagnosis of DM2 they were additionally reviewed by cardiologists who recommended they could continue to take the medicine with a yearly cardiac review.

\section{Case B: Runs in the family}

A 32 year old gentleman was referred to clinic with a history of muscle stiffness. Early childhood development was normal. He was active in primary school. Symptom onset was at age $12 . \mathrm{He}$ noticed stiffness and locking in his legs, hands and occasionally jaw and tongue. He enjoyed hockey, but was unable to keep playing as stiffness predominantly affected his legs (especially initiation of movement or sudden movement) and was particularly troublesome in the cold. Muscle symptoms had a tendency to improve as he warmed up.

Examination was notable for calf hypertrophy, gait and grip myotonia.

In terms of family history, his brother described similar symptoms but his parents and sister were unaffected.

Clinical history and examination was indicative of myotonia congenita and genetic testing identified a heterozygous c.654G>A, p. Gly285Glu mutation in the chloride channel gene CLCN1 confirming the diagnosis. This mutation is pathogenic and described in numerous unrelated families with myotonia congenita. The inheritance pattern in some, however, is autosomal dominant and in others autosomal recessive. Segregation testing was undertaken in the family. His asymptomatic mother and sister did not carry the mutation while his symptomatic brother did. His father, although 
asymptomatic and without any EMG evidence for myotonia, was also found to be heterozygous for the mutation.

All coding regions of the CLCN1 gene were sequenced and MLPA performed to exclude any gene rearrangements. Genetic analysis of the SCN4A gene and testing for DM1 and DM2 were also negative.

\section{What can be the inheritance pattern in myotonia congenita?}

\section{Autosomal dominant}

2. Autosomal recessive

3. De novo dominant

\section{Autosomal dominant with reduced penetrance}

\section{Any of the above}

Correct answer: 5

Discussion

\section{What is the inheritance pattern in the family?}

To further assess the effect of the Gly285Glu CLCN1 mutation, cell-based electrophysiological characterisation of the effects of the mutation in an oocyte model was undertaken. The mutation had definite effects on chloride channel function, and these effects are similar to those seen in other dominantly inherited CLCN1 mutations. In this family two individuals who were heterozygous for the mutation displayed clinical symptoms whereas their father was asymptomatic. In our diagnostic service the inheritance pattern seen in families with this variant is variable reflecting what is reported in the literature i.e. some appear dominant and others recessive. We excluded other scenarios e.g. concomitant DM2.

Overall, given two symptomatic family members who were both heterozygous for the mutation, previous descriptions of the CLCN1 Gly285Glu mutation in multiple dominant pedigrees, the cell electrophysiology compatible with dominant inheritance, and the lack of any confounding genetic 
variants in other genes, the inheritance pattern in this family was felt to be most consistent with dominant inheritance with reduced penetrance.

\section{CASE C: A treatment challenge}

A 15 year old boy was transitioned from paediatric services. He was born premature but reached developmental milestones well. At age eight, following a viral gastroenteritis, he developed weakness of arms and legs lasting 24hours. In subsequent years he had a similar episode once or twice per year but in the few years prior to transition this had escalated to two to three episodes per week. At the age of 14 , he was admitted to hospital with quadraparesis. Serum potassium was $2.5 \mathrm{mmol} / \mathrm{L}$. A diagnosis of hypokalaemic periodic paralysis was confirmed with a genetic mutation in CACNA1S (p.Arg528His). He was commenced on acetazolamide and attack frequency reduced to two to three per month.

His attacks of paralysis typically occur in the morning, on waking. They can be triggered by high carbohydrate intake. Occasionally, his hands become weak in the cold or he may have some weakness if exercising after a significant break.

At review on transition to the adult clinic, his symptoms had deteriorated again to two to three attacks per week, despite being prescribed acetazolamide $250 \mathrm{mg}$ three times daily. He reported however that due to commitments at school he would often forget the midday dose. At this stage, his acetazolamide dose was changed to a twice daily dose of 500mg, allowing for easier administration and a slight dose increase. He additionally commenced daily Sando-K (potassium supplementation) two tablets in the morning.

He enrolled in college, but on average would miss two days per week due to attacks. If he woke with weakness, he couldn't get out of bed until $2 \mathrm{pm}$. Amiloride $5 \mathrm{mg}$ daily, increasing to $5 \mathrm{mg}$ twice daily, 
was added to acetazolamide and Sando-K. This regimen, improved some severity of symptoms but not to a degree sufficient to prevent days off.

Amiloride was changed to spironolactone $50 \mathrm{mg}$ daily in an attempt to gain better control of the attacks but this was not tolerated due to side effects and was changed to epleronone. A combination of eplerenone, acetazolamide and daily sando-K reduced the duration and frequency of attacks although did not abolish them entirely.

His mother carries the same CACNA1S mutation and has reported symptoms since age 17. However, she has mild, infrequent episodes of weakness that do not significantly impair function or prevent work attendance and have not required treatment.

Which of the following is not a treatment for hypokalaemic periodic paralysis?

\section{Spirinolactone}

2. Acetazolamide

3. Bendroflumethiazide

4. Amiloride

5. Potassium supplements

Correct answer: 3

\section{Discussion}

Why was the proband more severely affected than his mother when they both have the same genetic mutation?

Reduced penetrance and variable severity of phenotype with the same mutation is relatively common in skeletal muscle channelopathies ${ }^{14}$. The same mutation can even cause different phenotypes within the same family (see also Case 2). This may be influenced by epigenetic factors and differential allelic expression ${ }^{15}$. Additionally it is reported that men are more severely affected by the periodic paralyses than women ${ }^{14}$. This was illustrated in the proband with severe, treatment 
resistant symptoms, while his mother with the same CACNA1S mutation is mildly affected not requiring any pharmacological treatment.

\section{What should the approach to treatment in hypokalaemic periodic paralysis consider?}

Due to the episodic nature of symptoms in the channelopathies with return to baseline, they are sometimes regarded as relatively benign. Frequent, prolonged and severe episodes however are severely limiting with additional disability or disadvantages accrued if unable to participate in education, socialisation (especially in teenage years) and work ${ }^{16}$. Frequency and severity of symptoms, detrimental effect on daily life/activities and any obvious triggers to symptoms are all factors to consider in the approach to management.

Identification and minimisation of triggers e.g. consumption of large carbohydrate loads, is useful in all cases - regardless of overall severity. Keeping a food diary can be a useful way to highlight foods that may be especially detrimental to an individual. Including the timing of meals is also helpful as many patients report the influence of carbohydrates in the evening is worse the less time they are taken before going to sleep.

Those with mild and infrequent episodes may prefer not to take daily medication. The majority of patients in our clinical experience require management with at least one agent although they may be more severely affected individuals by the nature of their referral to clinic. If pharmacological agents are considered Acetazolamide, a carbonic anhydrase inhibitor, is often used as first line treatment and can be effective in any form of periodic paralysis. In hypokalalemic periodic paralysis specifically, other agents aim to prevent low serum potassium e.g. potassium sparing diuretics such as amiloride or spironolactone which are taken daily as a prophylactic attempt to prevent attacks or reduce their frequency. Potassium supplementation is more rapid acting and frequently taken at the onset of an attack to try and reduce its duration and/or severity. A small regular daily adjunctive dose in conjunction with other prophylactic medications can also sometimes be beneficial. In difficult to treat cases a combination of agents may be required. 
The aim of therapy is ultimately to reduce the frequency, duration and severity of episodic symptoms. A subset of patients develop progressive fixed weakness but it is unclear as yet the extent that this relates to frequency of attacks and whether aggressive symptomatic treatment would prevent or reduce this.

More recently, Magnetic Resonance Imaging has been used to identify patients with significant STIR signal change in muscles. STIR signal suggests oedema related to an attack in these patients and may be a potential biomarker for treatment efficacy although this requires further exploration.

Other factors to consider in management include providing an emergency plan to the local team with instructions for administering IV potassium and cardiac monitoring in the event of a profound quadraparesis and significantly low serum potassium. Education regarding the condition and suitable adjustments should also be provided to school and work.

\section{CASE D: All muscle.}

An 18 year old boy was born at term with no perinatal complications. He was smaller than average at birth, but well and subsequent development was normal. He was sporty in primary school, with comparable abilities to his peers and enjoyed playing football. He had some dental abnormalities requiring an orthodontist's input for overcrowding and misalignment.

There was no significant family history. His parents and older sister were well.

At age 11 he collapsed at school. By-standers described sudden loss of consciousness and thought he had a fit. When an ambulance arrived, an unspecified cardiac ventricular arrhythmia was detected. On arrival to hospital, nodalol was commenced but did not control the rhythm. He was subsequently commenced on Flecainide and Verapamil with control of the rhythm achieved. Subsequent cardiac monitoring in childhood recorded transient ventricular tachycardias. At age 14, a reveal device was implanted to aid monitoring. 
Despite intermittent cardiac conduction abnormalities he did not report any palpitations or other cardiac symptoms. On specific questioning, he did notice a little weakness in his legs after exercise but had thought this was normal. He had no episodes of paralysis. On examination, he was of short stature with evidence of micrognathia, hypertelorsim and clinodactyly. Neurophysiology testing demonstrated a positive long exercise test. Genetic testing revealed a KCNJ2 mutation (c.368T>G, p.Val123Gly) consistent with a diagnosis of Andersen-Tawil syndrome.

In a patient presenting with suspected periodic paralysis, which test is most helpful in indicating a diagnosis of Andersen-Tawil syndrome?

\section{Creatine kinase}

2. An ECG

3. A muscle biopsy

4. Their height

5. Clinodactyly

Correct answer: 2

\section{Discussion}

\section{What is the cardiac spectrum of disease in ATS?}

ATS has historically been thought to have a generally benign cardiac phenotype with life-threatening arrhythmias occurring only rarely. The key features reported include presence of $u$ waves on the ECG (Figure 2) and ventricular ectopics ${ }^{17}$. However, there is increasing literature on the occurrence of more severe arrhythmias and the need for implantable cardiac defibrillators. In our cohort of 62 patients at the National Hospital for Neurology and Neurosurgery, $8 \%$ of those with genetically confirmed ATS have required an implantable cardiac defibrillator for sustained ventricular tachycardia or cardiac arrest. Additionally, $12.5 \%$ have cardiogenic syncope. Patients typically complain of few cardiac symptoms or only palpitations and without regular monitoring there is a risk 
serious arrhythmia will be undetected. We recommend all ATS patients (with or without cardiac symptoms) have at least yearly cardiac review including a minimum of $24 \mathrm{hr}$ holter monitoring.

\section{What characteristic features are seen in ATS and can they aid diagnosis?}

ATS is described as a triad of periodic paralysis, cardiac conduction defects and dysmorphic features but only two out of three are required for diagnosis. It is notable that the episodic muscle weakness in ATS is also often not profound or does not result in quadraparesis (as in our case) meaning this feature can be present but its significance overlooked. Similarly not all patient complain of cardiac symptoms or may only report occasional palpitations. An ECG is often abnormal however (Figure 1) and can be a useful investigation in anyone presenting with periodic paralysis, even if they don't appear dysmorphic.

Classically described dysmorphic features include short stature, hypertelorism, low set ears, micrognathia, small hands and feet, clinodactyly and syndodactyly ${ }^{5,6}$. However, in some patients these features may be subtle and certainly not all aspects are present in everyone. Some patients may be of normal height or even tall for their gender and age. In our practice, the most consistently present feature appears to be clinodactyly but this is a relatively common dysmorphic feature and not specific to ATS. This broad range of distinctive features and even the complete absence (or subtlety) of distinctive features is important to be aware of. The absence of these features should not dissuade clinicians from pursuing genetic testing for KCNJ2 mutations. 


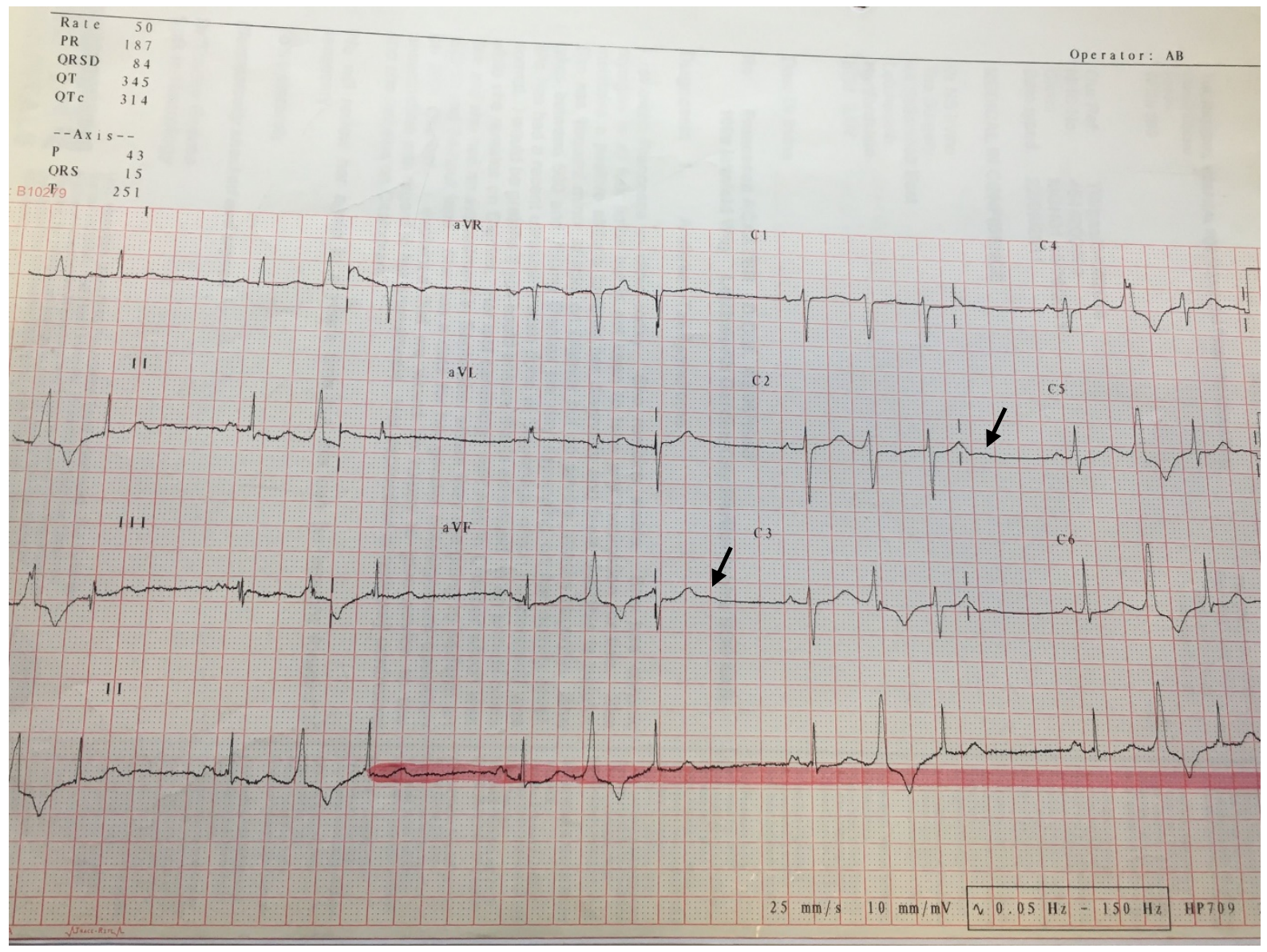

Figure 1: ventricular ectopics and U waves (black arrows) seen on a resting ECG in patients with

ATS.

\section{CASE E: Fatigue}

A thirteen year old boy presented with lethargy. He was born at term and development was normal.

At age nine, he noticed myalgia and limb pain which was exacerbated during an inter-current illness.

Despite recovering from the upper respiratory tract infection, he had persistent although variable

lethargy and pain, slept and ate poorly. He missed a substantial amount of school. At this stage,

routine investigations were normal with the exception of a slightly elevated for age Creatinine

Kinase of 305IU/L. He was diagnosed with chronic fatigue syndrome and symptoms persisted. There was no significant family history or other past medical history. 
Two years later, he woke with heaviness and weakness in his legs which progressed over the course of the day. He went out on a school trip but by that evening he was unable to walk or sit upright, had paralysis of all limbs and poor head control. He was taken to the emergency department where a serum potassium level of $2 \mathrm{mmol} / \mathrm{L}$ was recorded. This was corrected with oral potassium supplementation and the weakness subsequently improved over the following 48 hours. He had three further similar episodes over the next three months. The weakness was always worse in the mornings with some improvement in strength over the day. There were no identifiable triggers. Daily episodes of myalgia and heaviness in the legs continued. School attendance remained poor. On examination, there was no fixed muscle weakness, atrophy or hypertrophy. A long exercise test was normal. Creatinine Kinase was mildly elevated at 495IU/L. Genetic testing identified a pathogenic de novo CACNA1S mutation confirming a diagnosis of hypokalaemic periodic paralysis.

Subsequent treatment with potassium supplementation, acetazolamide and amiloride was effective in reducing the severity and frequency of weakness and fatigue as well as episodes of full paralysis. School attendance significantly improved.

Which of the following are not symptoms of hypokalaemic periodic paralysis?

\section{Episodic muscle weakness}

2. Fatigue

3. Syncope

4. Myalgia

5. Progressive muscle weakness

Correct answer: 3

Disucssion

Are the symptoms of fatigue and myalgia relevant? 
Fatigue and myalgia as the presenting symptom of periodic paralysis is rare, however a significant proportion of patients do describe fatigue and report that lack of energy is a significant aspect to the impact on quality of life ${ }^{18}$. The Independent Quality of Life questionnaire (INQoL) and SF-36 was administered to sixty-six patients with skeletal muscle channelopathies. Patients with myotonia congenita and hypokalaemic periodic paralysis had the worst perception of the quality of their life. Muscle weakness and fatigue was an important contributor to this perception.

When this patient was referred to us we felt in retrospect some of his fatigue and myalgia was accompanied by weakness and probably did reflect the onset of periodic paralysis symptoms but it was not until his presentation with quadraparesis that this became evident.

This case also highlights the impact of hypokalaemic periodic paralysis on educational potential. As attacks often occur first thing in the morning but can resolve by the afternoon they can also be misinterpreted as school avoidance. Liaising with the school to ensure adequate understanding of the condition, allowing pupils to attend in the afternoon even if they have missed the morning and sending school work to be done at home are all useful avenues that can be deployed to limit the impact on learning and achievement.

\section{References}

1. Horga A, Raja Rayan DL, Matthews E, et al. Prevalence study of genetically defined skeletal muscle channelopathies in England. Neurology. 2013;80(16):1472-1475.

2. Fontaine B, Khurana TS, Hoffman EP, et al. Hyperkalemic periodic paralysis and the adult muscle sodium channel alpha-subunit gene. Science. 1990;250(4983):1000-1002.

3. Ptacek $L$, George AL, Jr., Griggs RC, et al. Identification of a mutation in the gene causing hyperkalemic periodic paralysis. Cell. 1991;67(5):1021-1027.

4. Bulman DE, Scoggan KA, van Oene MD, et al. A novel sodium channel mutation in a family with hypokalemic periodic paralysis. Neurology. 1999;53(9):1932-1936.

5. Tawil R, Ptacek L, Pavlakis SG, et al. Andersen's syndrome: potassium-sensitive periodic paralysis, ventricular ectopy, and dysmorphic features. Ann Neurol. 1994;35(3):326-330. 
6. Andersen ED, Krasilnikoff PA, Overvad H. Intermittent muscular weakness, extrasystoles, and multiple developmental anomalies. A new syndrome? Acta Paediatr Scand. 1971;60(5):559564.

7. Matthews E, Fialho D, Tan SV, et al. The non-dystrophic myotonias: molecular pathogenesis, diagnosis and treatment. Brain. 2010;133(Pt 1):9-22.

8. Miller TM. Differential diagnosis of myotonic disorders. Muscle Nerve. 2008;37(3):293-299.

9. Fournier E, Arzel M, Sternberg D, et al. Electromyography guides toward subgroups of mutations in muscle channelopathies. Ann Neurol. 2004;56(5):650-661.

10. Suominen T, Schoser B, Raheem O, et al. High frequency of co-segregating CLCN1 mutations among myotonic dystrophy type 2 patients from Finland and Germany. J Neurol. 2008;255(11):1731-1736.

11. Furby A, Vicart S, Camdessanche JP, et al. Heterozygous CLCN1 mutations can modulate phenotype in sodium channel myotonia. Neuromuscul Disord. 2014;24(11):953-959.

12. Smith CA, Gutmann L. Myotonic Dystrophy Type 1 Management and Therapeutics. Curr Treat Options Neurol. 2016;18(12):52.

13. Groh WJ. Mexiletine is an effective antimyotonia treatment in myotonic dystrophy type 1. Neurology. 2011;76(4):409; author reply 409.

14. Venance SL, Cannon SC, Fialho D, et al. The primary periodic paralyses: diagnosis, pathogenesis and treatment. Brain. 2006;129(Pt 1):8-17.

15. Duno $M$, Colding-Jorgensen $E$, Grunnet $M$, Jespersen $T$, Vissing J, Schwartz $M$. Difference in allelic expression of the CLCN1 gene and the possible influence on the myotonia congenita phenotype. Eur J Hum Genet. 2004;12(9):738-743.

16. Matthews E, Silwal A, Sud R, et al. Skeletal Muscle Channelopathies: Rare Disorders with Common Pediatric Symptoms. J Pediatr. 2017;188:181-185 e186.

17. Zhang L, Benson DW, Tristani-Firouzi M, et al. Electrocardiographic features in AndersenTawil syndrome patients with KCNJ2 mutations: characteristic T-U-wave patterns predict the KCNJ2 genotype. Circulation. 2005;111(21):2720-2726.

18. Sansone VA, Ricci C, Montanari M, et al. Measuring quality of life impairment in skeletal muscle channelopathies. Eur J Neurol. 2012;19(11):1470-1476. 